Livraisons

d'Histoire

de l'Architecture

\section{Livraisons de l'histoire de l'architecture}

16 | 2008

Grands chantiers et matériaux

\title{
l'église Saint-Pierre de Firminy, de Le Corbusier : quarante ans d'histoire entre idée et réalisation
}

Le Corbusier Saint-Pierre's church in Firminy: a forty years history between idea and realization

Die Kirche Saint-Pierre, Firminy, von Le Corbusier : eine vierzigjährige

Geschichte zwischen Entwurf und Fertigstellung

\section{Luca Sampò}

\section{OpenEdition}

\section{Journals}

Édition électronique

URL : http://journals.openedition.org/lha/194

DOI : $10.4000 /$ /ha. 194

ISSN : 1960-5994

Éditeur

Association Livraisons d'histoire de l'architecture - LHA

Édition imprimée

Date de publication : 10 décembre 2008

Pagination : 153-172

ISSN : 1627-4970

Référence électronique

Luca Sampò, « l'église Saint-Pierre de Firminy, de Le Corbusier : quarante ans d'histoire entre idée et réalisation », Livraisons de l'histoire de l'architecture [En ligne], 16 | 2008, mis en ligne le 10 décembre 2010, consulté le 19 avril 2019. URL : http://journals.openedition.org//ha/194 ; DOI : 10.4000/Iha.194

Ce document a été généré automatiquement le 19 avril 2019

Tous droits réservés à l'Association LHA 


\title{
l'église Saint-Pierre de Firminy, de Le Corbusier : quarante ans d'histoire entre idée et réalisation
}

\author{
Le Corbusier Saint-Pierre's church in Firminy: a forty years history between idea \\ and realization \\ Die Kirche Saint-Pierre, Firminy, von Le Corbusier : eine vierzigjährige \\ Geschichte zwischen Entwurf und Fertigstellung
}

Luca Sampò

«L'image authentique du passé n'apparaît que
dans un éclair. Image qui ne surgit que pour
s'éclipser à jamais dès l'instant suivant. »
W. Benjamin, Sur le concept d'histoire, §v.

\section{L'église de Saint-Pierre}

1 L'église Saint-Pierre, projetée entre 1960 et 1965, est la dernière œuvre de Le Corbusier pour la petite ville minière de Firminy. Une base carrée, abri du centre paroissial, surmontée par un cône hyperboloïde au sommet tronqué diagonalement vers le sud, qui laisse pénétrer la lumière solaire par le haut. Géométrie simple aux symbolismes complexes, formes archétypiques qui plongent leurs racines dans une recherche de monumentalité aux contours «laïques»: crassier, pyramide, tour industrielle, mécanisme acoustique aux résonances cosmiques.

2 Le Corbusier y travaille pendant cinq ans, évaluant plusieurs solutions en dialogue étroit avec la paroisse locale mais, à sa mort, le projet demeure inabouti. Six ans après, en 1971, un chantier ouvre et, en 2006, l'église est achevée. Elle est inscrite aujourd'hui dans la continuité de l'œuvre corbuséenne: dernier projet sacré dont la paternité lui est attribuée, et dont la construction a été tout-à-fait posthume. 


\section{L'idée, l'atelier et les éléments de la méthode de travail}

Le premier croquis concernant l'église est daté du 4 juin 1961 : sur une seule feuille de son carnet $^{1}$, Le Corbusier résume un plan, une élévation et une coupe. Ce dessin, au graphisme sommaire, présente cependant toutes les composantes architecturales qui formeront le projet définitif. À l'atelier de la rue de Sèvres, le collaborateur chargé de suivre le projet est José Oubrerie. L'élaboration se déroule pendant trois ans, à travers un parcours de recherche complexe, constamment et dialectiquement vérifié avec l'association paroissiale de Firminy-Vert.

4 L'analyse complète des documents qui marquent ce parcours a permis de définir les linéaments de la méthode de travail de Le Corbusier ${ }^{2}$ : le point de départ de chaque phase du projet vient d'une idée qu'il transfère en dessin, soit une pensée reprise sur ses carnets, soit une correction des dessins d'atelier. Le projet est ensuite développé à l'atelier en plan, coupe et élévation. Chaque fois qu'il parvient à une définition suffisante, Le Corbusier en demande une maquette, qui constitue toujours la vérification finale d'une idée, et non un point de départ. Si une modification intervient ensuite, elle est suivie de dessins et d'une nouvelle phase du projet. Dans le cas de l'église de Saint-Pierre cet iter se répète cinq fois entre juin 1961 et la fin de $1963^{3}$, date des derniers plans connus concernant l'église, approuvés par Le Corbusier et enregistrés dans le «livre noir » de l'atelier".

Le projet touche au but en décembre 1963 : à cette occasion Le Corbusier précise que son « travail d'architecte est d'une exactitude totale. Il ne laisse aucun aléa. Il en est au stade des plans d'exécution $»^{5}$.

\section{Le débat autour du projet de référence}

6 En 1964 le diocèse renonce au projet ${ }^{6}$, Le Corbusier meurt en 1965, le chantier ouvre en 1971 et, en 1978, deux tiers du bâtiment sont construits ; en 2006, l'église est achevée.

7 Elle attire aujourd'hui l'attention d'un public plus vaste que celui que composent les architectes, les historiens et les spécialistes. La question qu'on va se poser autour du projet de référence est pourtant primaire, étant donné que l'achèvement aurait été fait «à l'identique ${ }^{7}$ ». 
III. 1 : Plans, élévations et coupe de l'église Saint-Pierre d'après la dernière série de dessins de l'atelier Le Corbusier de décembre 1963

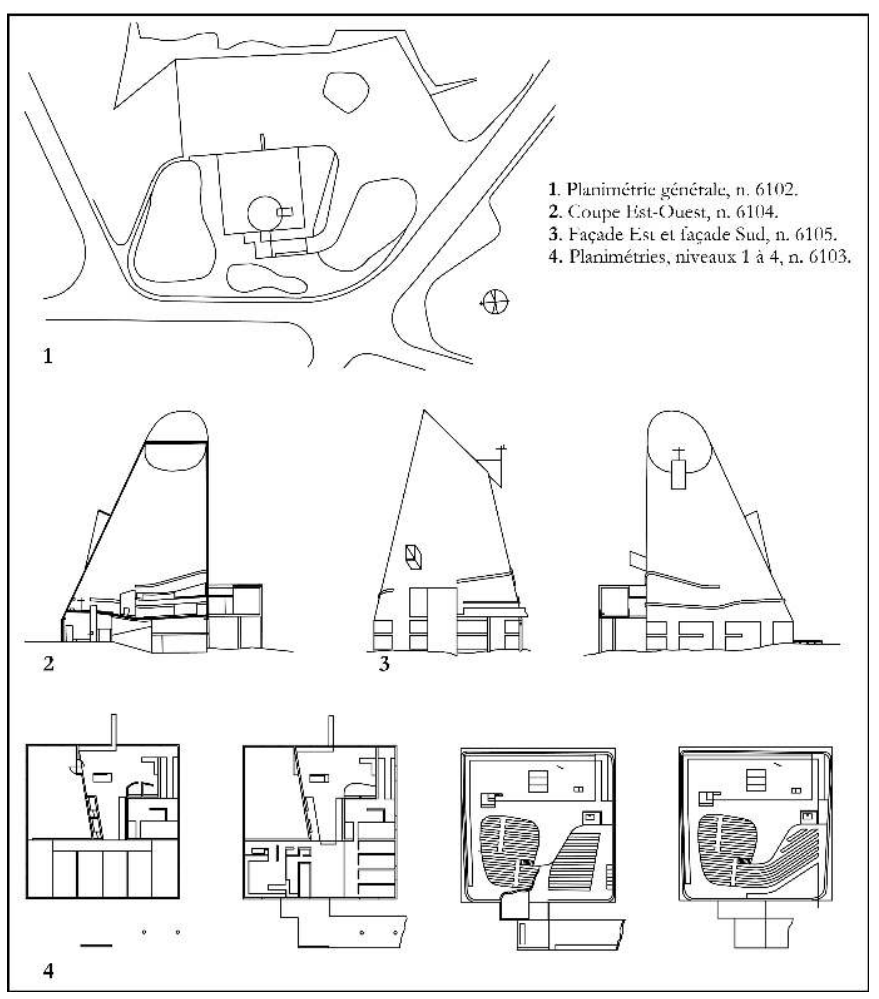

Cl. L. Sampò rédigés en atelier en décembre $1963^{8}(\mathrm{ill}$. 1964, que lui-même désigne dans une lettre envoyée au président de l'association paroissiale le 23 Janvier 1964 : «Vos plans sont terminés et signés par moi [...] Ne vous inquiétez pas; vos plans sont scrupuleusement étudiés et je crois que votre église sera une chose très importante ${ }^{9}$.»

âtiment est formé d'un "prisme carré de 24 mètres de côté portant une coque mince en béton armé de 36 mètres de haut de base carrée et de sommet constitué par une surface ovoïde inclinée portant le clocher ${ }^{10}$ ». La coque "hyperboloïque » est obtenue «par projection oblique d'un cercle situé au sommet sur la base carrée. Cette surface est ensuite coupée au sommet par un plan oblique qui donne une section proche d'un ovoïde, fermant la coque et portant le clocher ${ }^{11}$ ». L'étude de ces dessins a permis de définir les dimensions générales de l'église ${ }^{12}: 41$ mètres de hauteur totale, 25 au niveau bas de la dalle de couverture, dont l'inclinaison est d'environ $46^{\circ}$. Les quatre façades sont formées par des triangles reliés entre eux par des surfaces hyperboloïdes.

En 1968 - le projet ayant été repris en vue de l'ouverture du chantier - les dessins de Louis Miquel et José Oubrerie montrent toutefois une église formée par un carré de base ayant 25,5 mètres de côté et une hauteur totale de 35 mètres. La hauteur de la coque a été ainsi réduite de six mètres au sommet, et d'environ cinq mètres au niveau bas de la dalle ovoïdale de couverture. En conséquence, l'inclinaison de celle-ci passe de $46^{\circ}$ à $34^{\circ}$, en réduisant sensiblement l'élan du bâtiment (ill. 3). 
III. 3 : Élévations Sud-Nord de l'église d'après les projets de Le Corbusier, 1963, et d'Oubrerie, 1974-2002

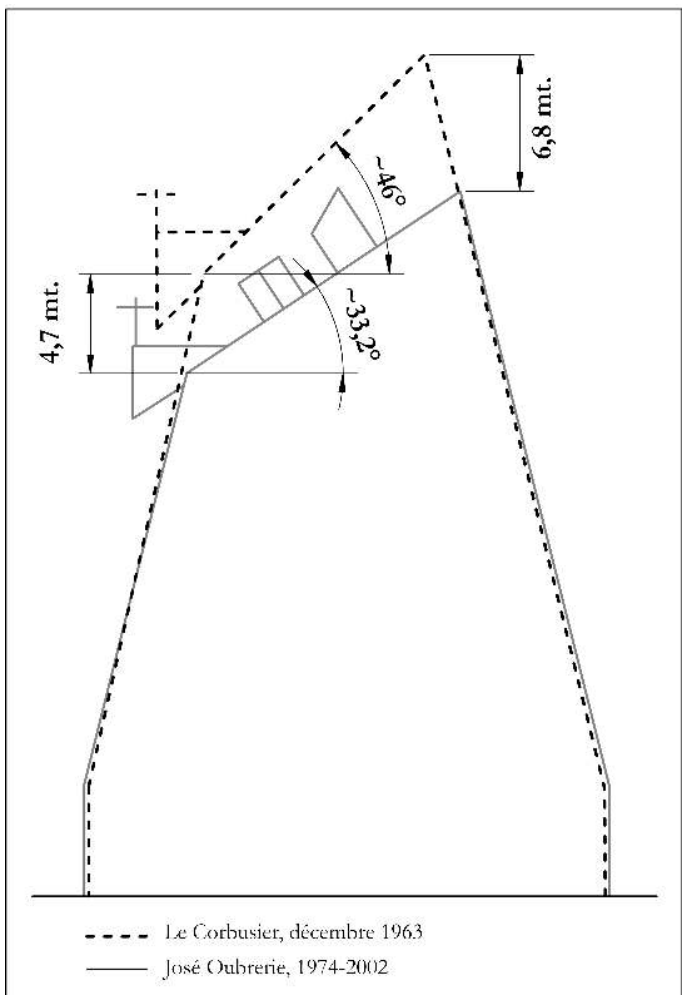

Cl. L. Sampò

11 Le changement de hauteur, par contre, ne modifie pas l'angle d'inclinaison des façades latérales, qui change très peu $-77^{\circ}$ à $76^{\circ}$ façades nord et sud, $65^{\circ}$ à $63,4^{\circ}$ façade est - grâce à un élargissement en surface de la dalle ovoïde (ill. 2, tableau 2).

Ill. 2: Tableaux récapitulatifs des variations dimensionnelles et des rapports proportionnels de l'église entre 1962 et 2002. Cl. L. Sampò.

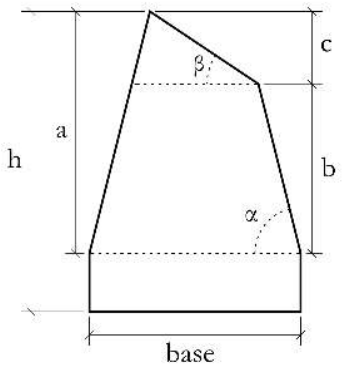

\begin{tabular}{|l|c|c|c|c|}
\cline { 2 - 5 } \multicolumn{1}{c|}{ Tablean 1 } & base & $\mathrm{a}$ & $\mathrm{b}$ & $\mathrm{c}$ \\
\hline Le Corbusier, 1963 & 0,7 & 1 & 0,7 & 0,3 \\
\hline Maquette Dirlick, 1964 & 0,83 & 1 & 0,73 & 0,27 \\
\hline Oubrerie, tracé regulateur, 1968 & 0,87 & 1 & 0,7 & 0,3 \\
\hline Miquel cı Oubreric, $\mathbf{1 9 7 0}$ & 0,88 & 1 & 0,68 & 0,32 \\
\hline
\end{tabular}

\begin{tabular}{|c|c|c|c|c|c|c|c|c|c|}
\hline Tablean 2 & base (mt.) & $\mathrm{h}$ (mt) & $\mathrm{a}(\mathrm{mt})$. & $\mathrm{b}$ (mt.) & $\beta$ & $a N$ & $\alpha E$ & as & $\alpha O$ \\
\hline Le Corbusier, mai-juillet 1962 & 21 & 48 & 39 & 25,7 & $49^{\circ}$ & $-81^{\circ}$ & $\sim 75^{\circ}$ & $-80^{\circ}$ & $-85^{\circ}$ \\
\hline Le Corbusier, novembre 1962 - juillet 1963 & 24,5 & 40 & 33,5 & 23 & $41^{\circ}$ & $77^{\circ}$ & $65^{\circ}$ & $77^{\circ}$ & $90^{\circ}$ \\
\hline Le Corbusier, décembre 1963 & 25,07 & 41 & 35,6 & 25 & $46^{\circ}$ & $77^{\circ}$ & $65^{\circ}$ & $78^{\circ}$ & $90^{\circ}$ \\
\hline Miqucl - Oubreric, 1968 & 25,5 & 35,3 & 28,8 & 20 & $34^{\circ}$ & $76^{\circ}$ & $63,7^{\circ}$ & $76^{\circ}$ & $90^{\circ}$ \\
\hline Miquel - Oubrerie, tracé regulateur, 1969 & 25,5 & 34,5 & 29,14 & 20 & $34^{\circ}$ & $76^{\circ}$ & $63,4^{\circ}$ & $76^{\circ}$ & $90^{\circ}$ \\
\hline Oubrerie, 1974 & 25,5 & 35,9 & 29,14 & 20,4 & $33,4^{\circ}$ & $76^{\circ}$ & $63,4^{\circ}$ & $76^{\circ}$ & $90^{\circ}$ \\
\hline Oubrerie, APD 1995 & 25,5 & 34,8 & 29,14 & 20,3 & $33,9^{\circ}$ & $76^{\circ}$ & $63,8^{\circ}$ & $76^{\circ}$ & $90^{\circ}$ \\
\hline Oubreric, APD 2002 & 25,6 & 34,2 & 29 & 20,3 & $33,2^{\circ}$ & $76^{\circ}$ & $63,8^{\circ}$ & $76^{\circ}$ & $90^{\circ}$ \\
\hline
\end{tabular}



construction baissant la hauteur de la coque n'y est explicitée ${ }^{18}$. Une note de José Oubrerie à l'attention de Le Corbusier, au sujet de la réunion du 8 juillet, ne fait pas état de cet argument ${ }^{19}$.

19 Si Le Corbusier évaluait encore la possibilité de baisser la hauteur de la coque pour réduire le coût de construction, pourquoi continuait-il à travailler - et à faire travailler les entreprises - sur le projet de 1963, faisant toujours référence à la série des dessins numérotée de 6102 à 6105, même après juillet 1964? Ces dessins sont à la base du descriptif et du métré des lots de février 1964, ainsi que du devis estimatif rédigé en avril et ils ont été envoyés à Claudius-Petit le 3 juin et aux entreprises le 8 juin suivant. Et encore, le 28 septembre 1964, après la réunion du 8 juillet, les mêmes plans sont appelés les « derniers plans d'architecture ${ }^{20}$ », et envoyés à l'entreprise Stribick afin d'effectuer une nouvelle estimation du coût de construction. 


\section{La reprise du projet : 1968-1978}

20

Eugène Claudius-Petit est, à la mort de Le Corbusier, le premier à manifester la volonté de voir bâtir l'église à Firminy-Vert, au même endroit envisagé auparavant avec Le Corbusier, dans l'ensemble du « centre civique » conçu par l'architecte. La fondation Le Corbusier ne parvient qu'en 1968 à assurer à Claudius-Petit qu'elle «suivra la progression de cette construction avec le plus grand intérêt et sera toujours prête à lui donner toute l'aide possible ${ }^{21} »$. Au lendemain de cette décision, le cardinal archevêque de Lyon donne son accord pour la construction de l'église et le diocèse est prêt à participer « en une certaine mesure » à la construction du bâtiment, « en esprit d'hommage à Le Corbusier et en souvenir de lui, [...] signe un protocole d'accord ${ }^{22} »$. Le projet est repris à l'étude en 1968, sous la direction de Louis Miquel et de José Oubrerie.

\section{Le tracé régulateur}

21 Entre juillet 1969 et janvier 1970, Oubrerie définit le "tracé régulateur ", qui sert à préciser les dimensions de l'église à partir d'un carré de base, et qui modifie substantiellement les rapports entre les trois dimensions principales de la coque. Suivant ce tracé, avec une base de 25,5 mètres de côté, la hauteur de la coque est de 29 mètres, sur une hauteur totale d'environs 35 mètres.

Au début de l'année 1969, Miquel et Oubrerie achèvent une première série de dessins dans lesquels le projet se montre déjà différent de celui de 1963. Entre-temps Oubrerie fait décaler l'emplacement de l'église d'un mètre vers l'est et d'un mètre vers le sud'².

En 1971 Claudius-Petit réussit à stipuler un marché officiel entre la Société civile, la ville de Firminy, l'entreprise Stribick, le diocèse, Miquel et Oubrerie en qualité d'architectes d'opération; marché clos sur la base d'une nouvelle série de dessins numérotée de 570 à 579,582 et 583 .

iocèse, toutefois, malgré l'accord de 1968, décide l'année suivante de renoncer définitivement à l'église, officiellement à cause du fait que le chantier n'était pas encore ouvert $^{24}$. D'autre part le diocèse avait été obligé de bâtir entre-temps un lieu de culte provisoire, en structure préfabriquée, utilisant une partie des fonds recueillis pour l'église de Saint-Pierre. En 1972 ce lieu de culte devenait largement suffisant aux besoins de la communauté donc, "d'un point de vue strictement "utilitaire", la construction de l'ensemble Le Corbusier [...] ne répond plus du tout à un besoin pour la paroisse ${ }^{25}$ ».

\section{Conception de la coque}

Entre 1970 et 1973, Miquel et Oubrerie poursuivent les études à la recherche d'ultérieures économies, d'un coté au niveau des fondations - en éliminant l'emploi des pieux profonds -, de l'autre au niveau de la couverture.

La coque engendrait un problème de coffrage à cause des surfaces hyperboloïdes qui reliaient les quatre façades de l'église. Des études sont menées afin d'en modifier la forme déplaçant le centre de gravité vers le milieu de la façade, mais surtout afin de transformer les surfaces hyperboloïdes en des arcs de cercle, de plus simple exécution. La courbe, les dimensions et les proportions de la dalle de couverture qu'Oubrerie avait définies en 
janvier 1970, et qui - sauf pour ce qui concerne l'adaptation aux nouvelles proportions du tracé régulateur - étaient à peu près conformes aux intentions de Le Corbusier, en seront profondément modifiées (ill. 4).

III. 4 : Axonométrie de la coque d'après le projet Le Corbusier, 1963, et bâtiment achevé, 2006

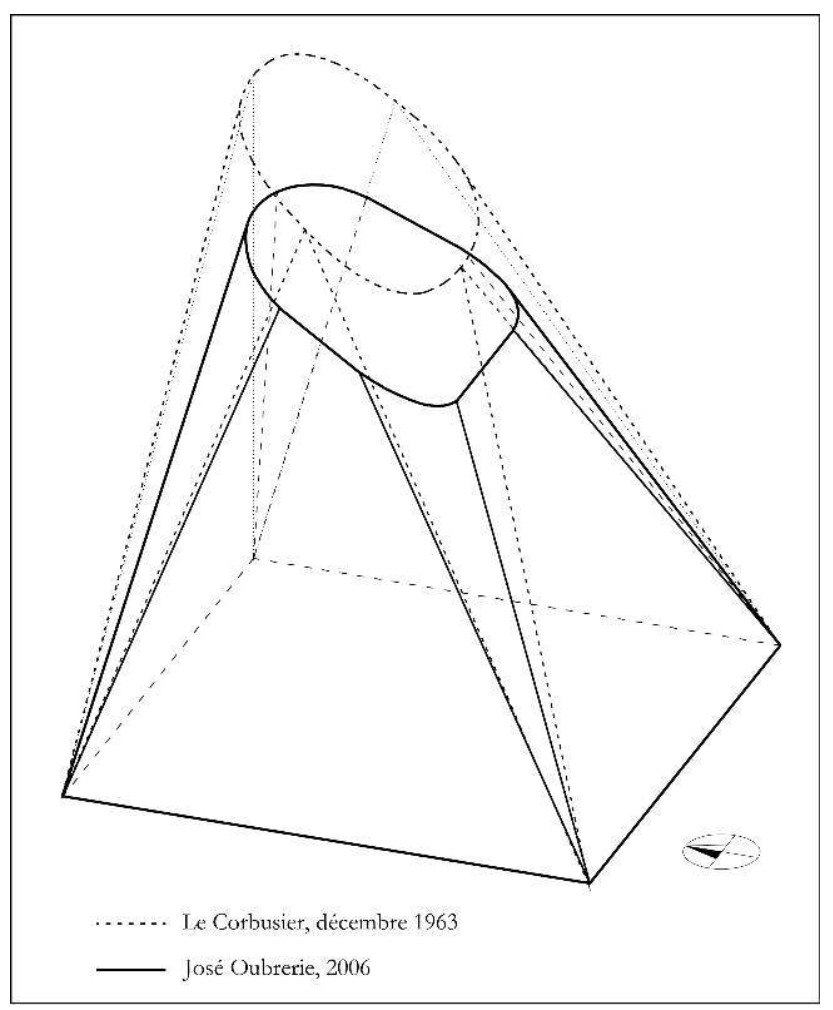

Cl. L. Sampò

Le 18 décembre 1973, l'entreprise Stribick parvient à une solution qui sera définitive : ils ont « fait réaliser une maquette de l'église ${ }^{26}$ » et ils assurent à l'entreprise Accetta « que le coffrage du fût de l'église pourra être réalisé comme prévu, et qu'il faudra le faire approuver par Monsieur Oubrerie ${ }^{27} »$. Dans le compte rendu de la réunion qui aura lieu le 19 décembre suivant : «La nouvelle construction du fût présentée par Monsieur Accetta est en principe acceptée par Monsieur Oubrerie. Base un carré de 25 mètres de côté, sommet un cercle parallèle en carré, le centre de gravité étant déplacé par rapport à celui du carré, ce qui a pour effet d'avoir pour raccordement dans les angles des cercles au lieu de paraboles comme dans le $1^{\text {er }}$ tracé d'où une plus grande facilité de coffrage..$^{28}$ ».

Par conséquent les façades est, ouest et sud sont transformées en surfaces trapézoïdales, la dalle de couverture, non plus une courbe ovoïdale continue, est composée, sur ces trois façades, par des courbes reliées par des lignes droites. L'image du bâtiment et la conformation même de la coque en résultent profondément altérée.

\section{Achèvement}

Étant donné le nombre important de modifications, petites et non et de nature différente, qui ont été apportées au projet, l'analyse sera centrée sur les éléments les plus significatifs du point de vue de la conformation de l'espace, et aussi du point de vue 
liturgique et fonctionnel. Cette étude ne prend pas du tout en considération les modifications des niveaux inférieurs du bâtiment, qui ont subi un changement d'usage ${ }^{29}$, et sont aujourd'hui destinées à des fonctions profondément différentes de celles qu'avait précisées Le Corbusier.

Les projets de référence qui ont été pris en considération et comparés dans ce travail sont la dernière version vérifiée et acceptée officiellement par Le Corbusier de décembre 1963, qui est sommaire dans les détails mais bien définie dans les composantes architecturales, les versions d'Oubrerie de 1970 et de 1974, l'APD de 1995, le projet d'achèvement de 2002, et l'œuvre achevée.

\section{Porche et entrée à l'église}

La rampe d'accès au porche de l'entrée principale de l'église se terminait, soit dans la version de 1963, soit dans la maquette d'Oubrerie de 1964, sous un auvent qui donnait lieu à un espace de "transition ", où le fidèle avait la possibilité de s'arrêter grâce à un banc en béton placé au nord. Cet espace était délimité par un mur qui empêchait à l'ouest la vue directe sur la ville, depuis l'église ${ }^{30}$ et donnait aux fidèles le temps de s'habituer à la lumière solaire en laissant pénétrer un éclairage transversal. Arrivant de la rampe, par contre, aucun mur ne délimitait l'espace derrière le banc laissant planer la vue sur l'horizon (ill. 5).

\section{5 : Porche d'entrée}

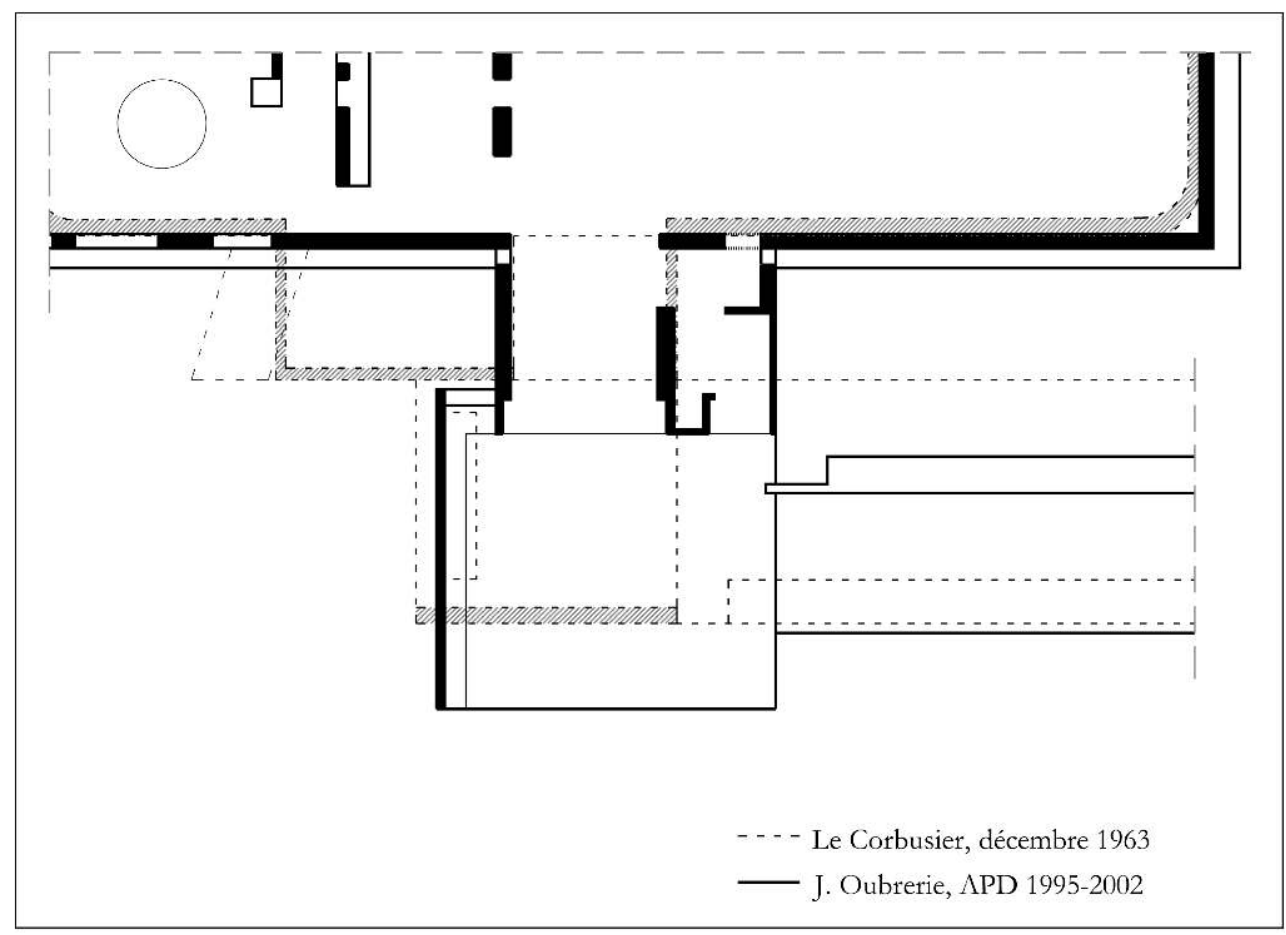

Cl. L. Sampò

Un deuxième espace de transition conduisait ensuite à l'église. Cet espace, plus sombre et délimité par des murs, permettait une transition douce entre l'extérieur et la nef. Il pouvait fournir soit un accès direct soit à l'église, soit au baptistère, soit à la «chapelle des morts ", répondant ainsi à une demande de la paroisse qui était précisée dans le 
programme remis à Le Corbusier en 1960 : «Baptistère : avec accès de l'extérieur et une porte d'entrée dans l'église. Chapelle funéraire : avec accès de l'extérieur si possible et accès direct de l'église ${ }^{31}$. Cet espace aurait du abriter aussi les fonts baptismaux, qui auraient dû être «bien situés dans leur fonction d'entrée dans l'église ${ }^{32}$ ». À partir des dessins de 1963, il est impossible de préciser la structure et la conformation de ces pièces, toutefois elles avançaient hors du périmètre de base vers l'ouest, en définissant ainsi un volume indépendant du porche et de l'église et soulignant la transition symbolisée par ces deux fonctions liturgiques de " passage ».

Ce volume indépendant disparaît dans le projet de 1970, l'espace correspondant est destiné à l'intérieur à "atrium revues ${ }^{33}$ ». L'arrivée de la rampe est disposée sous un auvent délimité par des murs sur les côtés nord et ouest, auxquels est adossé un banc en forme de « $\mathrm{L} »$, positionnant ainsi un plein, au lieu d'un vide, face aux fidèles venant à l'église. L'entrée se produit à travers un espace de transition délimité par une "grande porte » et une " petite porte ». Cette disposition, avec de petites variations et la réduction du banc au seul côté nord du porche, demeure inchangée jusqu'à l'achèvement.

\section{La chapelle du Patron}

La chapelle de semaine " trouve naturellement sa place en liaison avec le maitre-autel ${ }^{34}$ ", elle est placée à droite de l'entrée et au dessous du deuxième niveau de l'église. Cette liaison, que la paroisse avait expressément demandée, permet d'utiliser les places assises soit pour les célébrations dans la petite chapelle, soit pour celles se déroulant sur le maître-autel. Sa forme n'est arrêtée définitivement qu'en novembre 1963, et mise au point le mois suivant.

Les places assises étaient disposées selon une ligne droite inclinée vers l'extérieur au sud et selon une courbe, qui suivait le profil du plancher du niveau supérieur, au nord, sur une profondeur d'environ 9,5 mètres et une largeur maximale de 6,5 mètres. L'autel du saint patron était disposé au sud de l'axe de symétrie des places assises, d'importance mineure par rapport au maître-autel qui demeurait bien visible à partir de cette chapelle. L'autel, de petites dimensions, était abrité par deux murs en forme de « L » à l'est et au sud. Il était conçu pour un culte officié vers l'orient, selon les dispositions en vigueur avant le concile œcuménique de Vatican II.

À partir du projet de 1970, la conformation des places est transformée en trapèze, la surface est diminuée d'environ 16 mètres carrés, avec un côté parallèle à la façade sud de l'église et l'autre, en diagonale vers l'autel. La surface de la chapelle destinée à l'office du culte passe des 6,8 mètres carrés de 1963 aux douze de 1970. Cette disposition est abandonnée en 1974, à cause de l'insertion d'un escalier qui sert les places assises de la nef principale au niveau supérieur, derrière le mur qui abrite l'autel du saint patron. La présence de l'escalier provoque un déplacement important de l'autel vers l'ouest, et une petite réduction de l'aire destinée au culte en marge de laquelle est ajouté un poteau rond, de gros diamètre, qui réduit sensiblement la visibilité et l'effet de perméabilité de l'espace (ill. 6). 


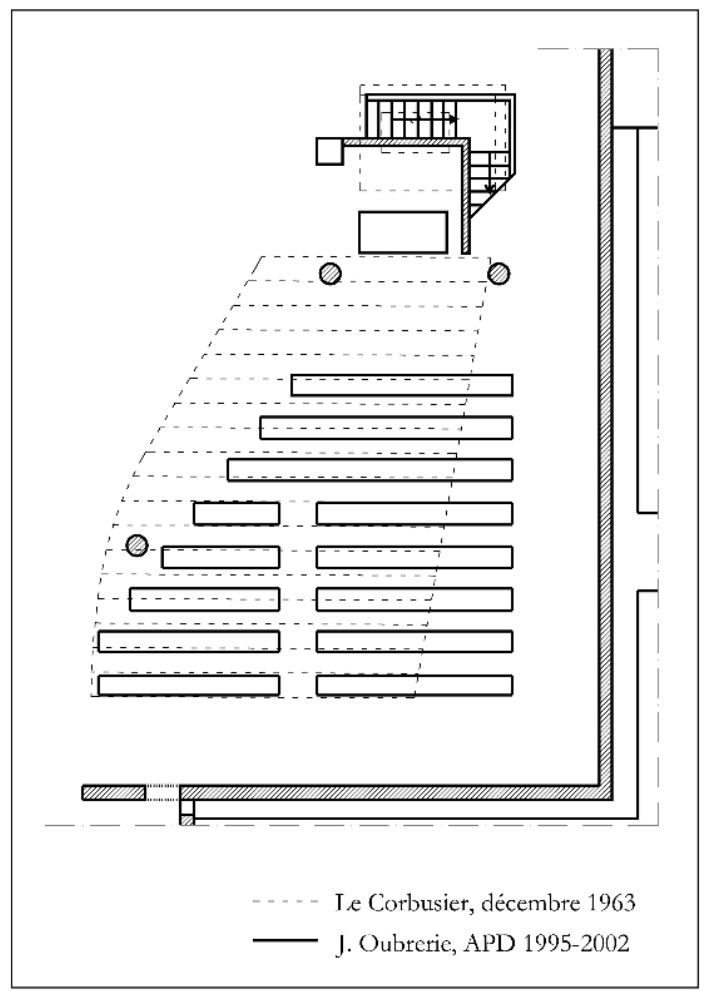

Cl. L. Sampò

En 1995, Oubrerie dispose l'autel de la chapelle de semaine en fonction du culte face aux fidèles, le déplaçant d'environ un mètre vers l'ouest. Cette disposition est, en substance, celle que l'APD de 2002 adopte, avec une réduction globale, pendant l'achèvement, des dimensions de la chapelle de 2,5 mètres vers l'ouest.

\section{Sanctuaire}

L'aire consacrée au culte accueille des fonctions différentes qui, au cours des décennies, ont plusieurs fois été réinterprétés. De forme rectangulaire à l'origine, elle est située à l'est, d'abord sur toute la façade, puis sur une surface moindre. Elle se rejoignait naturellement à la spirale du sol de l'église, qui monte vers le nord, sans différence de niveau avec la nef, alors qu'elle est surélevée à l'ouest par un gradin. En 1963, cette aire mesurait environ 21 mètres de largeur et 7 de profondeur, le maître autel était placé au centre et l'ambon - lieu de la parole - à sa droite; un banc en forme de « $\mathrm{L}$ » entourait toute l'aire et se prolongeait en dehors, jusqu'à la façade ouest. Le maître autel était au centre, sur l'axe de l'église, «à double fin, messes des deux côtés ${ }^{35}$ ». À gauche se trouvent la croix et un élément architectural décalé vers les fidèles, dont la fonction n'est pas précisée sur les dessins mais qui est présent déjà en novembre 1961, vraisemblablement une table de communion ou un siège pour le président.

L'ambon est composé du « lieu de la parole » proprement dit, surélevé symboliquement par des gradins, et d'un second appui en bas pour les autres lectures. Selon les indications de la paroisse et du père Tardy, il aurait dû occuper « une place vraie, bien visible, mais sans nuire à la place première de l'autel ${ }^{36} »$. Le Corbusier avait interprété cette indication 
en dessinant une structure de petites dimensions parallèle à l'autel principal et regardant directement les fidèles, qui dépassait d'un mètre la ligne délimitant le sanctuaire.

En 1970, le sanctuaire est surélevé dans toute sa longueur par rapport à la nef, le banc - maintenant en forme de « $\mathrm{U}$ » - est réduit à cette aire. L'élément à gauche du maître autel disparaît du dessin et, à sa place, une note indique simplement : «espace pour les baptêmes ${ }^{37}$ ». Le socle de l'autel majeur est augmenté d'un mètre dans sa largeur et derrière lui est ajouté un « siège du président ». L'ambon est ramené à la ligne délimitant le sanctuaire mais, si la structure basse demeure parallèle au maitre autel, le lieu de la parole est tourné de $17^{\circ}$ et regarde la salle diagonalement. Dans le projet de 1974 cette angulation est transférée à toute la structure de l'ambon, elle sera nouvellement modifiée dans l'APD de 1995 en assumant une importance majeure par rapport à la composition architecturale du sanctuaire et en s'enrichissant d'un «abat-son » derrière le lecteur. Cet abat-son ne figure pas auparavant dans les dessins d'Oubrerie ou Miquel ni dans le projet de Le Corbusier de 1963 mais une trace de sa conception se trouve dans un croquis de Le Corbusier de novembre 1962, sommaire dans sa définition, quoique très précis pour ce qui concerne la forme et la fonction de cette composante architecturale ${ }^{38}$. Dans l'APD de 2002 cette structure demeure essentiellement très proche à celle définie en 1995 : l'ambon est tourné de $8,5^{\circ}$ par rapport à l'axe principal du sanctuaire et la profondeur totale, sur laquelle sa structure est organisée, est devenue d'environ 4,6 mètres au lieu des 2,3 mètres carrés du projet de 1963 (ill. 7).

\section{7 : Ambon}

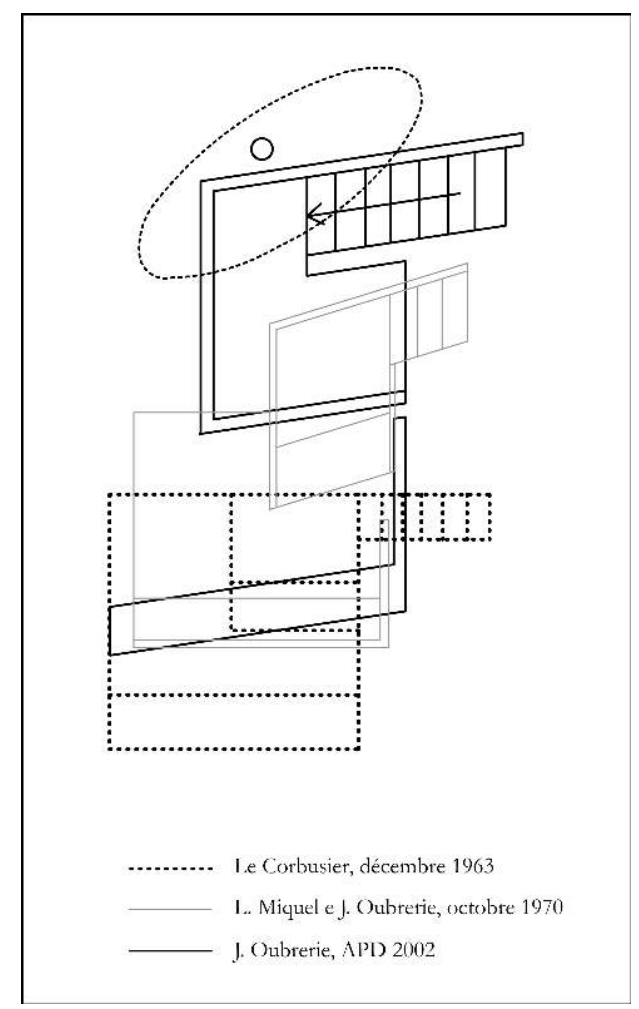

Cl. L. Sampò 


\section{Le plan en spirale de la nef}

41 La pente du plan en spirale, qui monte de l'entrée jusqu'au dernier niveau de l'église, ne subit que quelques modifications mineures du point de vue de l'inclinaison, ce sont plutôt la conformation spatiale et la disposition des bancs qui sont modifiés à partir de 1968. En 1974, après une première série de recherches pour définir la courbe de l'aire destinée aux bancs, le plancher supérieur est éloigné du sanctuaire d'environ un mètre et demi dans la partie sud, à cause de l'introduction d'un escalier qui le relie directement au niveau inférieur, et du décalage de la chapelle de semaine vers l'ouest.

Le Corbusier avait élaboré pour la disposition des bancs une solution conforme au programme de la paroisse, qui voulait des bancs « assez "ramassés" autour de l'autel, sans cependant de vis-à-vis des fidèles entre eux $^{39}$ ", et surtout qui permettaient une visibilité totale de l'autel majeur, à partir de n'importe quelle place. Dans cette disposition les bancs du quatrième niveau n'étaient pas alignés au plancher. En 1970, la disposition des bancs au quatrième niveau subit une transformation importante : les bancs sont alignés à la courbe du plancher, tournant d'environ $21^{\circ}$ vers le nord, et cette disposition oblige les fidèles - qui se trouvent face à l'ambon - à se tourner vers la droite pour regarder le maître autel (ill. 8).

\section{8 : Nef de l'église, sol en spirale et disposition des bancs}

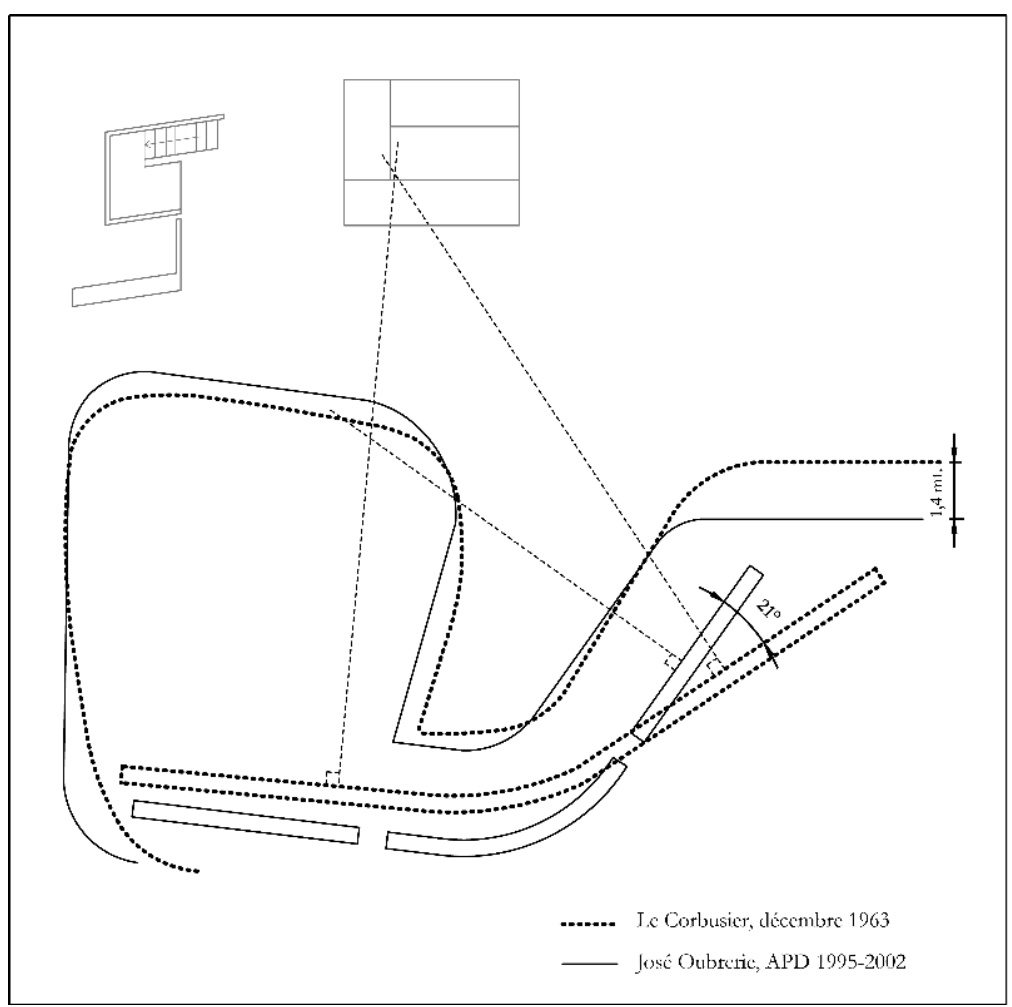

Cl. L. Sampò 


\section{Autres éléments}

Dans le projet de Le Corbusier, la liaison entre les niveaux inférieurs de la paroisse et la nef principale était assurée par une rampe qui, en 1970, est partiellement modifiée avec l'introduction de marches longues, n'ayant qu'une petite hauteur. En 1974, cette structure est remplacée par un escalier. Ce changement parait être justifié en relation au renoncement au projet de la part du diocèse et en conséquence aux modifications d'usage des étages inférieurs (ill. 9).

III. 9 : Le système de liaison verticale entre le centre paroissial et la nef de l'église, de gauche à droite : Le Corbusier 1963, Oubrerie 1970, Oubrerie arrêté 1974

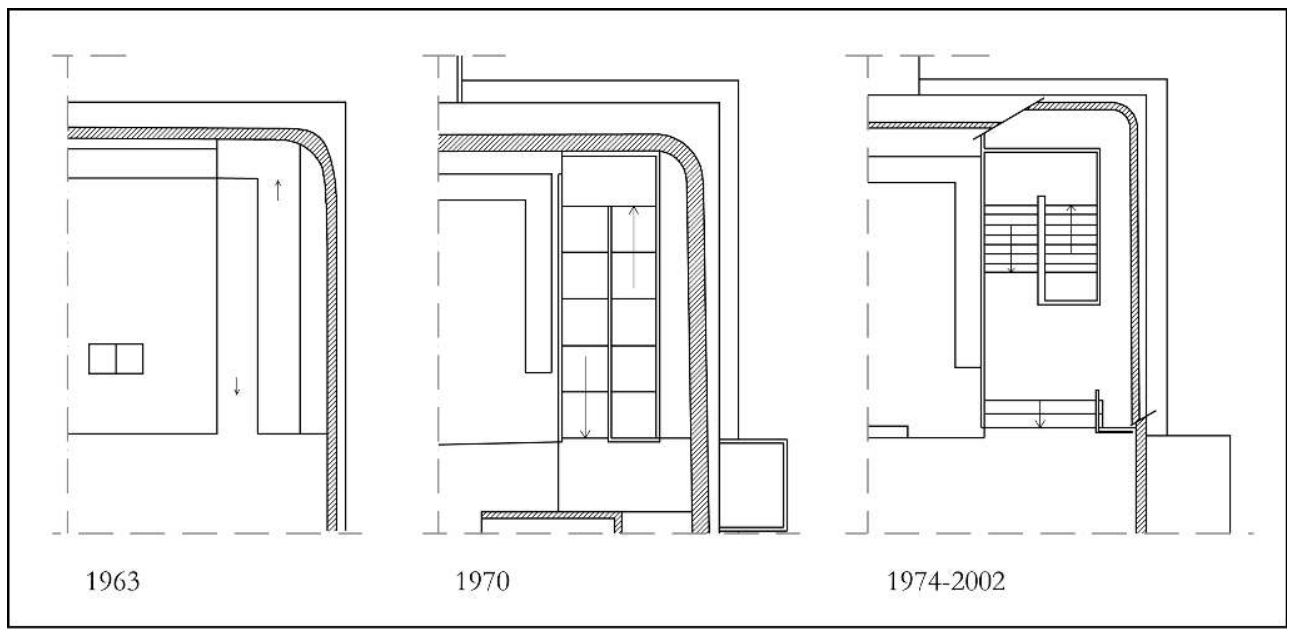

Cl. L. Sampò

À ce propos, Le Corbusier affirmait qu'une rampe «est une sensation totalement différente de celle donnée par un escalier formé de marches. Un escalier sépare un étage de l'autre : une rampe relie $e^{40} »$. L'escalier souligne et ratifie donc un passage qui a marqué profondément la vie de ce bâtiment jusqu'à son achèvement.

Au côté opposé de la rampe, se trouve l'escalier de secours, ajouté sur la façade nord en 1974. Auparavant, à sa place, Oubrerie et Miquel avaient prévu en 1970 un bassin de récolte des eaux pluviales. L'escalier est repris d'autres éléments du langage corbuséen et imite celui de la maison de la culture. Sa structure et ses dimensions ont changé dans le temps : en 1974, il montait vers l'ouest, dans l'avant projet détaillé de 1995, il est inversé vers l'est et un bassin pour la récolte des eaux pluviales de dimension importante est ajouté au nord; cette structure est modifiée encore en 2002 et, en cours de réalisation, avec l'insertion d'une pyramide rappelant celle de Ronchamp (ill. 10). 


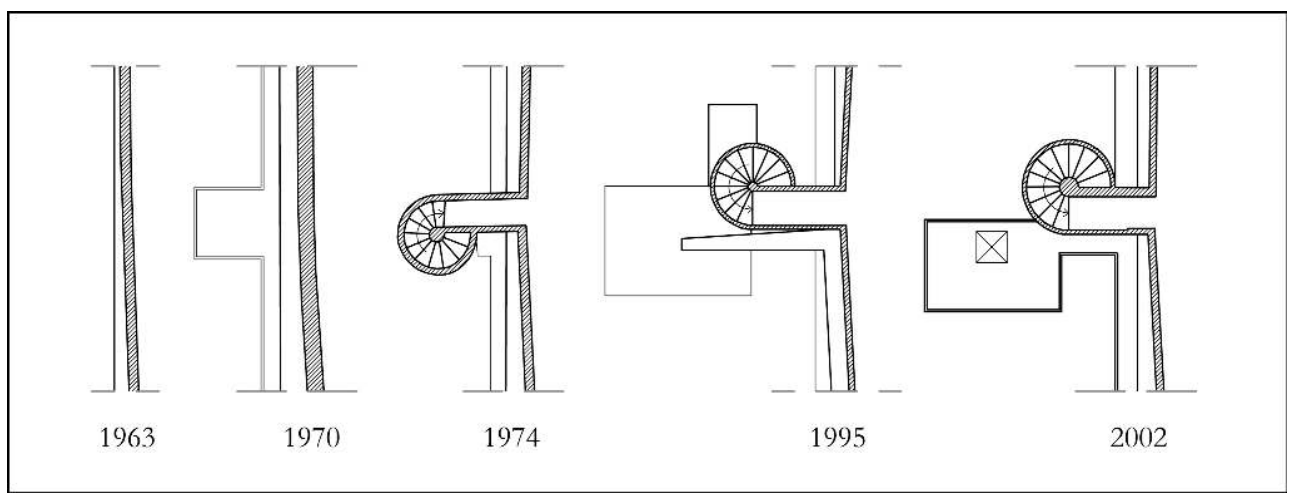

Cl. L. Sampò

Le mur constellé derrière le maître autel est présent à partir des premiers croquis de Le Corbusier en juin 1961 de forme circulaire. Toutefois, dans les dessins de décembre $1963^{41}$ sa forme et ses dimensions ne sont pas spécifiées. Dans le descriptif de février 1964, on indique tout simplement que « la face est, la plus inclinée, est raidie par une voile en béton perpendiculaire à la coque qui, en même temps, protège de la pluie les trous fermés par des verres de couleurs qui donnent une lumière diffuse au-dessus du sanctuaire ${ }^{42}$ ». L'indication des couleurs, bien que sommaire, confirme une note précédent de Le Corbusier selon laquelle la constellation aurait dû être " une claustra ouverte dans le ciment et faite de verres de couleurs diverses ${ }^{43}$ ». L'indication d'une "claustra » semble, par contre, faire allusion à une conformation architecturale qui n'est pas celle des «trous » comme à Ronchamp, mais une ouverture chantournée et répartie à l'intérieur d'un périmètre géométrique, de forme peut-être circulaire selon un dessin à déterminer, puis fermée par des verres colorés. La constellation choisie pendant les travaux d'achèvement - entre 2003 et 2006 - est celle d'Orion, mais aucune couleur n'est employée.

Aucune couleur n'était spécifiée non plus pour les trois canons à lumière, seul l'emplacement de celui sur la façade ouest avait été défini sur les dessins de 1963 et dans le descriptif de 1964 : « Le canon de section rectangulaire donne la lumière solaire du soir sur l'autel. Il est en béton et une dalle de verre y est enchâssée ${ }^{44}$. » Pour ce qui concerne la dalle ovoïdale de couverture, elle «est trouée par deux canons donnant la lumière zénithale ${ }^{45}$ ». Les deux canons en couverture étaient présent dès les premiers croquis de Le Corbusier, en 1961, où il avait noté que le premier aurait du être un «oculus minuit Noël » et l'autre un « oculus Pâques (heure exacte) ${ }^{46}$ ». Un rappel aux " fêtes cosmiques » -solstice d'hiver, équinoxe de printemps - dont il parlait souvent se référant au palais de l'assemblée de Chandigarh, des fêtes qui auraient dû rappeler aux êtres humains «la fraternité des rapports entre cosmos et êtres vivants. Étoiles, nature ${ }^{47}$ ». Ces deux canons sont insérés pour la première fois sur la maquette Dirlick de 1964, repris après dans les projets d'Oubrerie et Miquel de 1970 et 1974. L'avant-projet détaillé de 1995 montre un emplacement inversé par rapport aux précédentes, pendant que dans l'avant-projet détaillé de 2002 ils ont retrouvé un emplacement proche de celui du projet de 1970. 


\section{Mémoire et identité}

À propos de l'église Saint-Pierre, bien avant son achèvement, la fondation Le Corbusier assurait que «indéniablement, ce projet, lorsqu'il sera achevé, s'inscrira dans la continuité de l'œuvre de Le Corbusier ${ }^{48} »($ ill. 11).

III. 11 : L'église Saint-Pierre, Firminy, 2006

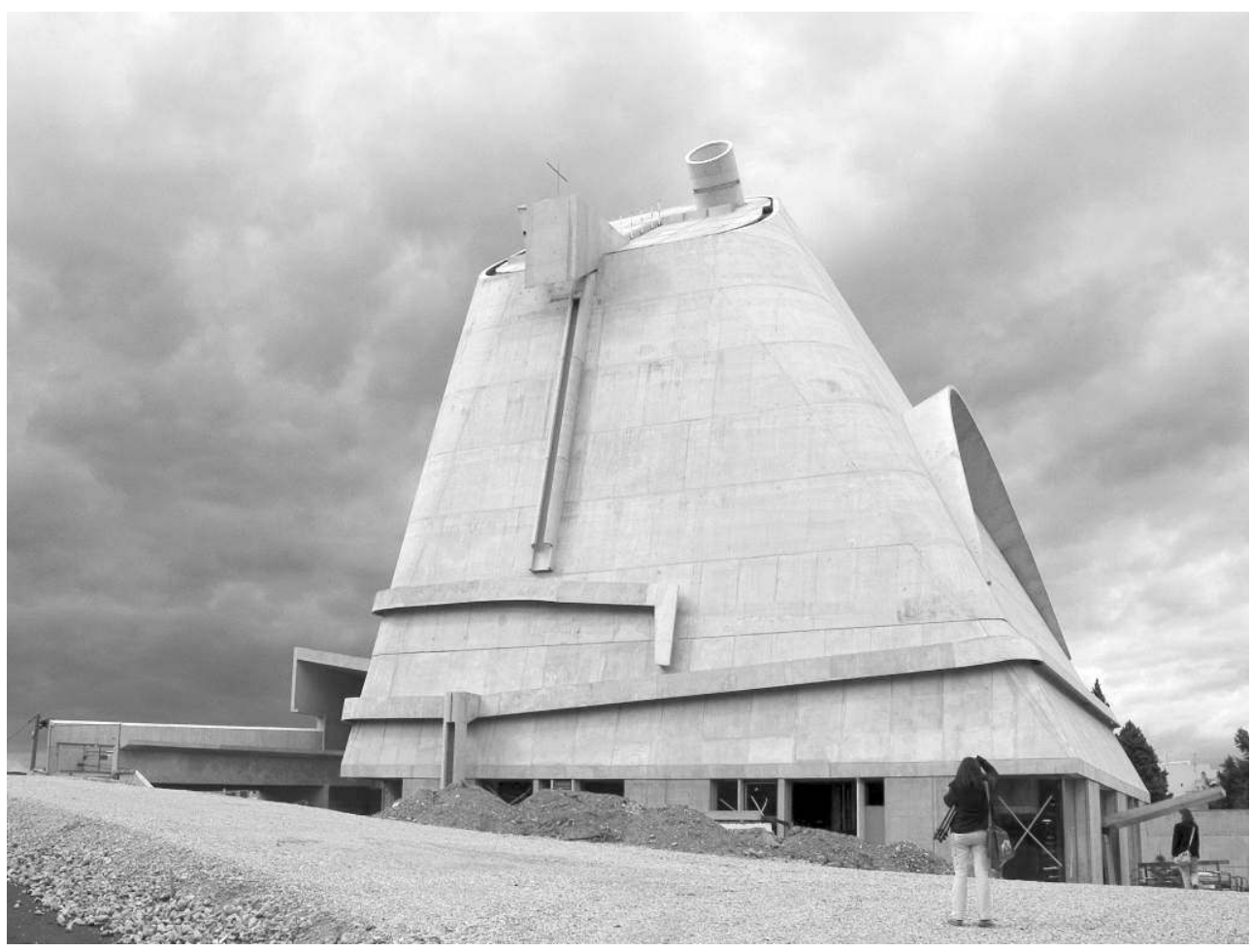

Cl. L. Sampò

Le patrimoine des bâtiments de Le Corbusier dans l'agglomération de Saint-Étienne est, évidemment, très important $\mathrm{du}$ point de vue touristique, économique et culturel. La commission supérieure des monuments historiques, réunie en mars 2003 « afin de donner toutes les recommandations et tous les conseils qui lui paraîtraient justifiés dans le cadre des travaux de restauration du bâti comme dans celui de la finition du projet ${ }^{49}$ ", considère elle même que, parmi les raisons en faveur de l'achèvement de l'église, figurent « une raison fonctionnelle (église et centre culturel), une raison économique (intérêt touristique $)^{50} »$, confirmant ainsi que «la culture vaut d'abord comme instrument de communication politique ${ }^{51} »$.

Il s'agit donc d'une «création » de patrimoine, mais la création d'une œuvre « ne suffit pas à produire du patrimoine. Encore faut-il que l'héritier accepte le legs du testateur. Ce legs fait donc l'objet d'un tri, résultat d'une double opération politique et scientifique ${ }^{52} »$. Qu'en sera-t-il de l'identité du bâtiment quand les générations futures auront vite oublié l'iter entier du projet ? Est-il légitime de demander le statut de monument corbuséen pour ce bâtiment ${ }^{53}$ ? "Le passé réclame une rédemption ${ }^{54}$ ", écrit Walter Benjamin, à chaque instant le présent le réclame aussi. 
51 Les modifications que le projet a subies après la mort de Le Corbusier jusqu'à son achèvement sont nombreuses et importantes, la conformation même du bâtiment a été en partie révolutionnée, le contexte urbain est changé, il a évolué au cours de quarante ans et il continuera à évoluer bien au delà du moment présent. L'image de patrimoine qui est en train d'être construite à Firminy ne pourra pas être fixée. L'église de Saint-Pierre est ainsi en passe de devenir le symbole d'un lien avec un passé qui n'a jamais existé, et qui est en train d'être re-construit.

\section{NOTES}

1. . Le Corbusier, croquis du 4 Juin 1961, carnet R 64 «Turin Mai 1961 », p. 33, archives de la Fondation Le Corbusier (dorénavant FLC) W1-4-749.

2. Recherches conduites entre 2003 et 2007 dans le cadre du doctorat en cotutelle entre l'École pratique des hautes études de Paris et l'université de Rome La Sapienza. L'étude est contenue dans la thèse de doctorat en histoire de l'art, section des sciences historiques et philologiques, École doctorale "histoire, archéologie, linguistique", sous la direction de M. Jean-Michel Leniaud, intitulée Le Corbusier 1957-1965. Traguardi di una ricerca teorica, artistica ed architettonica. Il complesso di Firminy, soutenue le 16 Juillet 2007.

3. . Les dessins d'atelier permettent d'identifier cinq phases différentes : 1er juin - septembre 1961 ; 2 octobre 1961 - février $1962 ; 3$ mai - juillet 1962; 4 novembre 1962 - juillet 1963; 5 décembre 1963.

4. Dessins de l'atelier Le Corbusier numérotés 6102, 6103, 6104 et 6105, datés du 23 décembre 1963, rédigés par José Oubrerie, vérifiés par Le Corbusier et inscrit dans le «livre noir» de l'atelier, montrant l'implantation de l'église dans l'environnement, les plans des quatre niveaux du bâtiment, une coupe est-ouest et les élévations sud et ouest. FLC n 16526, 16528, 16529 e 16530.

5. . Lettre de Le Corbusier à Jean-François Baud, président de l'association paroissiale, du $1^{\text {er }}$ février 1963, FLC N1-2-25.

6. . Le renoncement de 1964 est lié officiellement au coût de construction du bâtiment trop élevé. Les sondages $\mathrm{du}$ sol en fondation avaient indiqué la nécessité d'employer des fondations spéciales. Le diocèse propose à Le Corbusier de formuler un nouveau projet pour un site différent de sa propriété, éloignant l'église de l'ensemble du centre civique conçu par l'architecte. Le refus de Le Corbusier de déplacer l'église suscite l'abandon du diocèse.

7. . Archives du ministère de la culture et de la communication, direction de l'architecture et du patrimoine, sous-direction des monuments historiques et des espaces protégés, dossier d'inscription aux monuments historiques de l'église de Firminy, 42_Firminy, cote 42095-2-002, notes.

8. . Cf. n. 4 .

9. . Lettre de Le Corbusier à Jean-François Baud, président de l'association paroissiale, du 18 Janvier 1964, FLC U1-19-79 et N1-2-42.

10. . Le Corbusier, Description de l'église paroissiale Saint-Pierre de Firminy-Vert, du 10 Février 1964, pp. 9, p. 1, FLC N1-2-90.

11. Ibid.

12. Seul le côté de base, bien qu'il soit indiqué être de 24 mètres, mesure 25,05 mètres. 
13. . «Un projet d'église paroissiale de Le Corbusier », dans L'Art sacré, novembre-décembre 1964, n. 3-4, p. 2-24, p. 19-21. Ces images ont été ensuite republiées dans Le Corbusier, « Il progetto e gli appunti di taccuino per la chiesa di Firminy-Vert », dans Chiesa e Quartiere, n. 34, giugno 1965, p. 28-38. Pour ce qui concerne les maquettes il s'agit des mêmes clichés de L'Art sacré, voir la lettre de Giuliano Gresleri à Le Corbusier du 2 Avril 1964, FLC U1-19-119.

Et, beaucoup plus tard, dans A. Eardley (sous la direction de), Le Corbusier's Firminy Church, The Institute for Architecture - Urban Studies and Rizzoli International, New York, 1981.

14. . Le seul témoignage direct nous parvient de l'association Le Corbusier pour l'église de Firminy-Vert. Dans leur « Chronologie du dernier compromis Le Corbusier concernant la hauteur de l'église ", on indique que la maquette d'Oubrerie avait été demandée pour la réunion de juillet 1964. Une analyse approfondie de cette chronologie a été faite par Gilles Ragot dans le Rapport sur l'achèvement de l'église de Saint-Pierre, 2 vol., v. 1, non publié, 55 p., p. 19, archives FLC.

15. . Le père Maurice Cocagnac o.p. a été, avec le père Capellades o.p., co-directeur de la revue L'Art sacré de 1954 à 1969.

16. . Lettre de Eugène Claudius-Petit au père Cocagnac du 16 Juillet 1964, FLC dossier « correspondance avant 65 ».

17. . Livre sur lequel étaient enregistrés tous les dessins et les maquettes exécutés à l'atelier de la rue de Sèvres, FLC S1-16.

18. . Compte rendu de la réunion du 8 Juillet 1964, FLC U1-19-102 : «M. l'abbé Tardy est venu voir L-C à l'Atelier, accompagné de M. Portafaix (?), pour discuter avec lui l'étude éventuelle d'une nouvelle église à Firminy-Vert. Il a laissé deux plans ».

19. . Note manuscrite de José Oubrerie du 8 Juillet 1964, FLC U1-19-103: « Note pour Mr Le Corbusier - église de Firminy. Il faudra écrire (ou téléphoner) à Mr Pommet au sujet des fondations (et d'une aide du ministère de la culture). Vous en avez parlé l'autre jour (jeudi dernier ) avec Malraux et Claudius-Petit. »

20. . Lettre de Fernand Gardien à l'entreprise Stribick du 28 septembre 1964, FLC U1-19-178.

21. . Procès verbaux du conseil d'administration de la fondation Le Corbusier du 28 mars 1968, archives FLC.

22. . Note d'information donnée par "l'association paroissiale Saint-Pierre de Firminy-Vert», du 27 janvier 1974, 4 p., p. 2, archives de la maison de la culture "Le Corbusier de Firminy" (dorénavant $\mathrm{MDC}$ ). Les termes généraux de l'accord sont les suivants: «L'asssociation Le Corbusier décide de réaliser le projet Le Corbusier sous sa responsabilité et à sa charge ; la ville de Firminy mettra le terrain choisi par Le Corbusier à la disposition de la Société civile ; la paroisse prendra à sa charge le petit aménagement intérieur et le mobilier, dont elle conservera la propriété ; l'édifice sera mis à la disposition de la communauté catholique locale, selon des conditions d'usage à déterminer par la suite. »

23. . Oubrerie demande ce déplacement afin d'éloigner l'église de la piscine qui venait d'être achevée, sur dessin d'André Wogenscky.

24. . «Le 14 octobre 1972, 4 ans et 1/2 après l'accord de 1968, le chantier de l'église Le Corbusier n'étant toujours pas ouvert, l'évêque de Saint-Étienne écrit au président de l'association Le Corbusier: "À mon très grand regret, je suis obligé, en ce qui nous concerne, de considérer comme dépassé et caduc notre protocole d'accord du 12 Mai 1968. » Note d'information [...], op. cit., n. 20 , p. 2.

25. . Le diocèse décide de verser 100.000 francs à la Société civile pour la construction de l'église. Une somme «forcément arbitraire » qui tenait compte aussi du fait que parmi les dons recueillis certains «avaient pu être faits spécialement parce qu'il s'agissait de construire une église "Le Corbusier" ». Note d'information [...] op. cit., n. 20, p. 3-4.

26. . Note interne de l'entreprise Stribick du 18 décembre 1973, MDC EG-FIR 1-5 [N_I] 1.

27. Ibid. 
28. . Compte rendu de la réunion du 19 décembre 1973 au bureau Accetta, MDC EG-FIR 1-4 [C_R] 7, 2 p., p. 1.

29. Ces niveaux étaient initialement étudiés en fonction des nécessitées de la paroisse. Y étaient prévus des salles de catéchisme, des salles de réunion, une sacristie, les locaux du presbytère, etc. Les locaux ont été profondément remaniés après le renoncement du diocèse et ensuite en 2002 en fonction des nécessités du nouveau gestionnaire des espaces, Saint-Étienne métropole, ils seront affectés par une antenne permanente du musée d'art moderne de Saint-Étienne.

30. Il faut noter qu'à partir de ce point, le panorama est bloqué par le centre commercial et les immeubles de Firminy-Vert.

31. . Programme sommaire, Paroisse de Saint-Pierre de Firminy-Vert, remis à Le Corbusier par le père Tardy le 19 juin 1960, FLC N1-2-1.

32. . Eléments de programme pour l'église Saint-Pierre de Firminy-Vert, du 11 Février 1961, FLC U1-19-9, 3 p., p.2.

33. . José Oubrerie et Louis Miquel architectes, Église "Le Corbusier", projet du 27 octobre 1970, échelle $1 / 50^{\mathrm{e}}$, plan $\mathrm{n}^{\circ} 573$, niveau 4 , numéro 19.

34. . Un projet d'église [...], op. cit., n. 13, p. 20-21.

35. . Eléments de programme [...], op. cit., n. 30, p. 2.

36. . Ibid.

37. . Oubrerie et Miquel, Église [...], op. cit., n. 31, numéro 15.

38. . Croquis de Le Corbusier du 21 novembre 1962, FLC 16561.

39. . Eléments de programme [...], op. cit., n. 30, p. 2.

40. . Le Corbusier et Pierre Jeanneret, CEuvre complète 1929-1934, VIII, v. 2, Girsberger, Zürich, 1964, p. 25

41. Dessin $n^{\circ} 6104$, coupe est-ouest, atelier Le Corbusier, rédigé par José Oubrerie du 23 décembre 1963, FLC 16528.

42. . Le Corbusier, Description de l'église [...], op. cit., n. 10, p. 4.

43. . Le Corbusier, notes manuscrites au croquis du 18 Juin 1961, FLC 16608.

44. . Le Corbusier, Description de l'église [...], op. cit., n. 10, p. 4.

45. Ibid.

46. . Le Corbusier, notes manuscrites du 11-12 Juin 1961, carnet R 64 « Turin - Mai 1961 », p. 44, FLC W1-4-758.

47. . Le Corbusier, notes manuscrites, carnet E 20 « Bogotà - Mai 51 », p. 54-55, FLC W1-2-449 et 448.

48. . «Firminy (France), achèvement de l'église Saint-Pierre », dans Bulletin d'informations de la fondation Le Corbusier, n. 25, fondation Le Corbusier, Paris, mai 2004, p. 1.

49. . Rapport de la commission supérieure des monuments historiques, section travaux, séance du 3 mars 2003, Firminy (Loire): église Saint-Pierre : Restauration des substructions et réalisation des élévations, 11 p., p. 2.

50. . Ibid., p. 8.

51. . Jean-Michel Leniaud, Vingt-cinq ans d'histoire $d u$ patrimoine, dans Philippe Poirrier - Loïc Vadelorge, Pour une histoire des politiques du patrimoine, série « Travaux et documents $n^{\circ} 16 »$, comité d'histoire du ministère de la culture - fondation Maison des sciences de l'homme, Paris, 2003, p. 33-44, p. 35.

52. Ibid., p. 41.

53. . Une proposition d'inscription de l'église de Saint-Pierre et des autres bâtiments de Le Corbusier à Firminy dans l'inventaire des monuments UNESCO a été présentée.

54. . W. Benjamin, Sur le concept d'histoire, § ii, dans Gianfranco Bonola - Michele Ranchetti, Walter Benjamin. Sul concetto di storia, Einaudi, Torino, 1997, p. 64. 


\section{RÉSUMÉS}

L'église de Saint-Pierre à Firminy, dernier projet inabouti de Le Corbusier dans la ville, a été achevée et inaugurée en novembre 2006. Elle s'insère ainsi dans le prestigieux contexte des œuvres religieuses de l'architecte, à côté de Ronchamp et la Tourette. D'après l'examen des documents d'archive et l'étude de l'œuvre construite - achevée "à l'identique » - on trace ici les linéaments de l'iter de réalisation du bâtiment à partir du dernier projet de Le Corbusier. Objet de l'analyse sont plus spécialement les transformations subies par des éléments qui sont fondamentales dans l'idée corbuséenne du projet: la coque couvrante la nef, hauteur et inclinaison; les éléments liturgiques et architecturales qui conforment l'espace religieux; parcours d'accès et espaces de transition.

Pendant quarante ans le projet a évolué, le contexte aussi ; l'église de Saint-Pierre est ainsi en passe de devenir le symbole d'un lien avec un passé qui n'a jamais été, et qui aujourd'hui est en train d'être re-construit.

The Saint-Pierre Firminy's church, one of the lasts Le Corbusier's unrealized projects, was completed and inaugurated in November 2006. It stands now as the third sacred project of the architect, besides Ronchamp and La Tourette. Based on the analysis of archive's documents and realized building - achieved à l'identique - this article aims to define the main lines of the church construction iter, starting from the last Le Corbusier design. The study pays particularly attention to the transformation of those elements considered fundamentals in Le Corbusier's original idea: the cone covering church's nave, height and inclination; liturgical and architectural elements defining sacred spaces and spatiality; access path to the church and transitions spaces.

The project during forty years evolved, as the context; the church of Saint-Pierre is becoming the symbol of a link with a past that has never been, and that today it's about to be re-defined.

Die Kirche Saint-Pierre, nach dem letzten unvollendeten Entwurf von Le Corbusier in der Stadt Firminy endlich fertiggebaut und im November 2006 eingeweiht, lässt sich neben Ronchamp und La Tourette in das weltberühmte religiöse Werk des Architekten einfügen. Die Studie, die sich auf Archivdokumente stützt, nimmt sich die Untersuchung des nach den Planungsvorgaben des Architekten «à l'identique» fertiggestellten Werkes vor. Sie definiert die Grundzüge des Weiterbaus der Kirche mit Rücksicht auf Le Corbusiers Enwurf und zeigt, dass in seiner Auffassung grundsätzliche Elemente verändert worden sind: konische Schale, Höhe und Neigungswinkel des Schiffes, liturgische und architektonische Elemente des Kirchenraumes sowie Zugangs und Übergangsräume. Innerhalb von vierzig Jahren haben sich also Projekt und Kontext entwickelt, so dass die Kirche Saint-Pierre heute von einer Vergangenheit zeugen will, die nie existiert hat und einfach nachgebaut wurde. 


\section{AUTEUR}

\section{LUCA SAMPÒ}

Luca Sampò, né en 1976, est architecte et docteur de l'École pratique des hautes études. Sa thèse, Le Corbusier 1957-1965. Traguardi di una ricerca teorica, artistica ed architettonica. Il complesso di Firminy , a été soutenue sous la direction du professeur Jean-Michel Leniaud en 2007. Docteur en histoire et restauration de l'architecture à l'Université de Rome "La Sapienza", il a été membre de la rédaction de la revue Materiali e Strutture. Problemi di conservazione du département d'histoire de l'architecture, restauration et conservation des Monuments historiques de l'université "La Sapienza" de 2005 au 2007. Il est l'auteur d'articles relatifs à l'histoire de l'architecture contemporaine et en particulier à l'œuvre de Le Corbusier (1887-1965) : « Esecuzione 'differita' e restauri lecorbusieriani : dati tecnici e problemi aperti », dans Materiali e Strutture. Problemi di conservazione $n^{\circ}$ 3-4, 2004, n.s. (III), année II, p. 140-163 ; « Le Corbusier : la chiesa di Saint-Pierre a Firminy, quasi una inaugurazione / Le Corbusier : Saint-Pierre de Firminy church, nearly an inauguration ", dans Metamorfosi. Quaderni di architettura, n 62, septembre-octobre 2006, pp. 70-71. Il est actuellement enseignant-chercheur en histoire de l'architecture contemporaine à l’Université de Rome “La Sapienza”. Adresse électronique : luca.sampo@gmail.com. 